\title{
SIMULATION OF I-V STEPS SHARPENING FOR JOSEPHSON JUNCTIONS
}

\author{
T.F. Refai and L.N. Shchata ${ }^{*}$ \\ Faculty of Engineering, Dept. of Physical \& Mathematical \\ Engineering, Ain Shams University, Cairo, Egypt. \\ * Atomic Energy Establishment, Dept. of Mathematics \\ and Theoretical Physics, Cairo, Egypt.
}

\section{Abstract:}

The effect of $r f$ frequency on the induced I-V steps for Josephson junction is discussed. The resistively shunted junction is applied for the case of an applied rf rectified sinsoidal current in which the amplitude is modulated by a saw toothed function. It is shown that the step rise sharpens on increasing the $r f$ frequency. We also show that the first fundamental step is nearly absent for low rf frequencies, while on increasing the $r f$ frequency, the step evolves and assume its well known configuration.

\section{Intraductions}

There has been considerable interest to invostigate the phenomena associated with Jasephann functions due to their potential use in superconducting quantum interference devices (SQUD'S), high speed ligic and memory devices, mixer madulators, and ac-amplifiers. One such afea of interest, is the induced steps in the I-V charbcteristics of a Jasephson junction driven by either an ac- or dc-curent sources of both. The effoct of critical current amplitude modulation(1), radio and micro wave power, end thetmal noise (thermal current)(2-5) an the induced steps has been widely investigated through the use of sevetal mathematical madels. In figute ( 1 ), the effect of varying the $t f$ frequency on the $I-V$ characteristics is shown(6). This figure shows scveral interesting features that occurs an increasing the if frequency. Such features are, the evolution of the first step, higher steps are better evalved than the first step, but still their step rise shatpens, end linally the ovet all curve rise incresses (i.e. approaching the ohmic line faster). It is to the best of our knowledge that such phenomeria has not been investigated and presented elsewhere.

Hence, it is the purpose of this paper to investignte the effect of if frequency an the features discussed above, fram the theoretical point of view. In ouf wark we applied the resistively shunted junction madel(7) (RSJ), for a Josephson junction biased by an rf rectified sinsoldal current phase relation, in which its amplitude is modulated by a sew toothed function. The madel is worked for three if frequencies 
$(10,50,100 \mathrm{MHz})$ at zeco temperature and for an if frequency $(10 \mathrm{MHz})$ at a notmslized tomperature $(t=0.75)$. It turns out the celculated $1-V$ curves agree very well (on a qualitative agreement basis) with the $1-V$ cutves of ligure (1). In sec. 2 the model is discussed for the zeto and non zero temperature, together with the sirrwalstion tectrique used. In sec. 3, the thearetical resusts are presented, discussed and compared to the experimental resulks Finally in sec. 4 we ptesent some closing canclusians.

\section{Theary:}

The response of a Josephson junction ta an ac current sautce $I_{B C} \sin e v t, \omega /$ is the applied external trequercy), while biased by a de current source is given by:

$$
\begin{aligned}
& c d V / d t+\sigma V+I_{c} \sin \phi=I_{d c}+I_{a c} \sin \omega t+I_{N}+ \\
& 2 e V=h d \phi / d t,
\end{aligned}
$$

Whete $\mathrm{C}$ is the Josephson function capacitance, $\mathrm{V}$ is the valtage acrosa the junction, junction conductance, I junction critical current, $\phi$ is the phase difference across the junction, and $I_{N}$ is the thermal cutrent generated in the resistor $R=1 / 0+$ at a given temperaturs. In eq. (1-b), $\hbar$ is Planck's constant divided by $2 \pi$, and $e$ is the electron charge. It is warth nated that eq. $(1-a)$, (fot $\left.\mathrm{F}_{\mathrm{N}}=0\right)$, is applicable to other physical systems such es the driven damped pendulum, a charged deraity wave in the presence of a time varying field pinned to an underlaying lattice, and a charged particle moving in a viscous medium dtiven by a periodic potential. In our work we will only consider the case for $C=0$ and $I_{d c}=0$. The tf curtent source will be replaced by:

$$
I_{0} \frac{t}{T}|\sin \omega t| \text {, }
$$

where (T) is the modulation periad of the sawteothed function $\left(\frac{T_{0}}{T}\right)$, and $I_{0}$ is the current amplitude at $t=T$. Next, by setting $u=t$ equation $(1-b)$ can be expressed as

$$
v=\frac{\hbar u r}{2 s} \frac{d \varphi}{d u}
$$

r ram $(1-8)$ and $(3)$, we then have:

$$
\frac{d G}{d u}=\frac{2 e R I}{\hbar} c\left[\frac{i_{0}}{T} u \sin u+i_{N}=\sin 0\right]
$$

where $i_{\mathrm{u}}$ and $\mathrm{I}_{\mathrm{N}}$ are the $\mathrm{rf}$ and thermal currents nermalized with respect to the critical current $t_{c}$. For the thermal current, the average distribution is given by: 


$$
\begin{aligned}
& \langle i\rangle_{\mathrm{rms}}=\left(\frac{\mathrm{k}_{\mathrm{B}} \mathrm{T}^{f}}{\mathrm{I}_{\mathrm{c}}^{2} \mathrm{R}}\right)^{1 / 2} \\
& \Delta t=\frac{2 e R \mathrm{I}_{\mathrm{c}}}{\hbar}(2 \Delta \mathrm{u})^{-1}
\end{aligned}
$$

Where $k_{B}$ is Goltzmarn constant, and $u$ is the normalized time step involved in the numerical integration of equation (4).

Equation (4) was solved numerically by applying the Runge Kutta method. Since our aim was to understand the qualitative nature of the solution, and not to get higher precisians, which would necessitate much longer complutation, $\mathrm{i}_{0}$ was taken up be $10^{\prime}$, and $T=10^{-3} \sec$ for all three frequencies (10, SD, $100 \mathrm{MHz}$ ), and $v=/ 100$. The normalized valtage is given by

$$
\langle v\rangle=\left\langle\frac{v}{R I}{ }_{c}=\frac{\hbar \omega}{2 e R I_{c}}\left\langle\frac{d v}{d u}\right\rangle\right.
$$

Where the averaging is teken over half the If cycle, which is also the case for the curtent. Finally its worth noting that for the if curtent, keeping $T$ constant and changing the frequency, has the sarne effect of keeping $w$ constant and varying $T$.

\section{Discussiont}

Figure (2) shows the numerical $1-\mathrm{V}$ curves at zero temperature and three If frequencies, 10,50 and $100 \mathrm{MHz}$. There are twa regions of interest in all curves. The first is that part of the curve that lies below a normalized curtent equal to two, which is the main interest of this peper. The sacond region is that for normalized currents values above two. In the first regian it is clearly shown how the first step exalves with increasing if frequency. In figure (3), the inset shows a generals step shape induced by an applied if current. On the path OA several If cycles elapse before $\mathrm{v}$ teaches $\mathrm{v}$. On increasing the curfent the system will move fram $A$ to $C$, where the duration between transitions decreases. At point $C$ the phase transition occurs evety cycle. Beyond paint $C$, the transition occuts evety few cycles on both the positive and negntive of swings At point B the transition rate isnow twice every cycle, and the system moves upward to point D at which a second step eppears(5). Bearing this in mind, we nowe pay our ottentions to figute (3), in which we placed the $10^{5} \mathrm{MHz}$ curve, (curve 1), elong side that of the $100 \mathrm{MHz}$ if trequency, (curve II), and try to reach a physical interpretation of how the first step is evolved. On increasing the current from 1 to $i=i_{1}$, the voltage on curve $I$ is $v_{1}$ (point A) which is less than $v_{2}$ (point B) for curve II. 


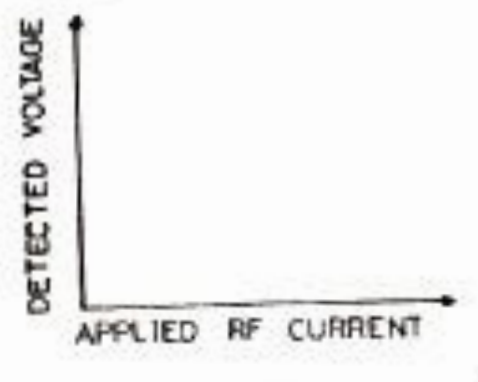

$69 \mathrm{MHz}$

$55 \mathrm{MHz}$

$50 \mathrm{MHz}$

$40 \mathrm{MHz}$

$35 \mathrm{MHz}$

$30 \mathrm{MHz}$

Figure (1): Experimental (1) $t-V$ curves for effferent if frequencies.

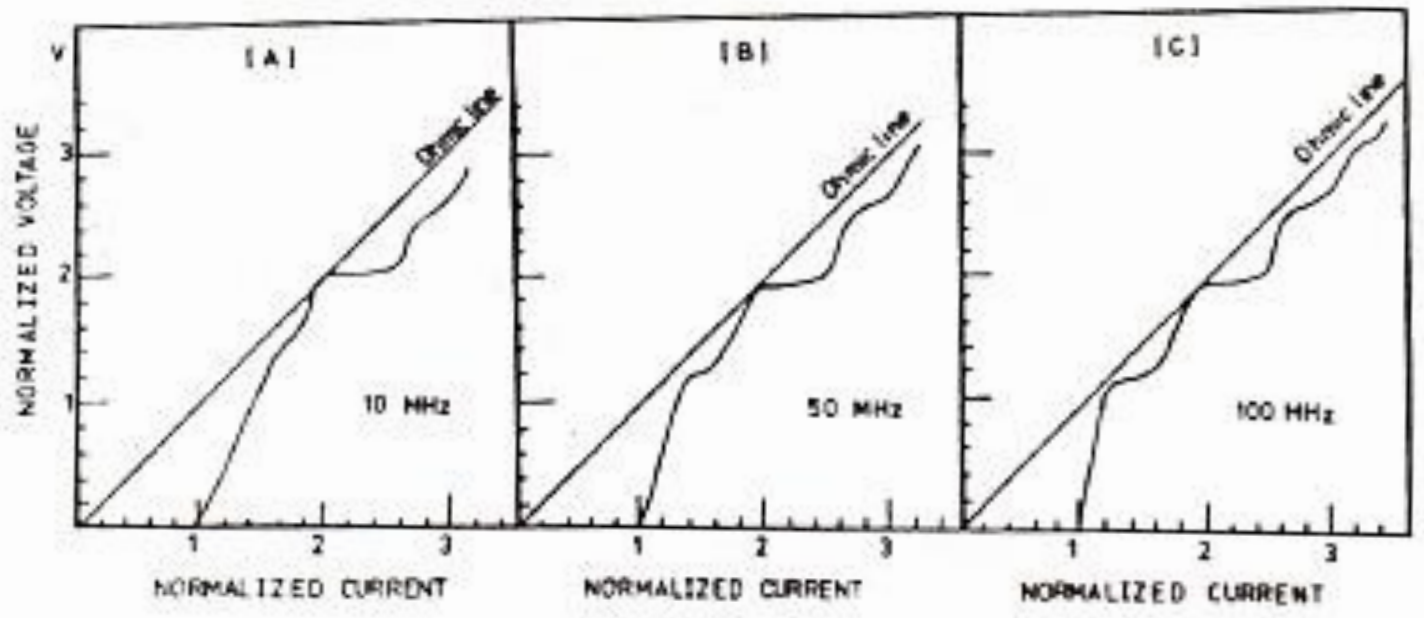

Figpure (2) Calculated $1-V$ curves for frequencies (a) $10 \mathrm{MHz}_{1}$ (b) $50 \mathrm{MHz}$ and (C) $100 \mathrm{MHz}$ at zern temperature. 


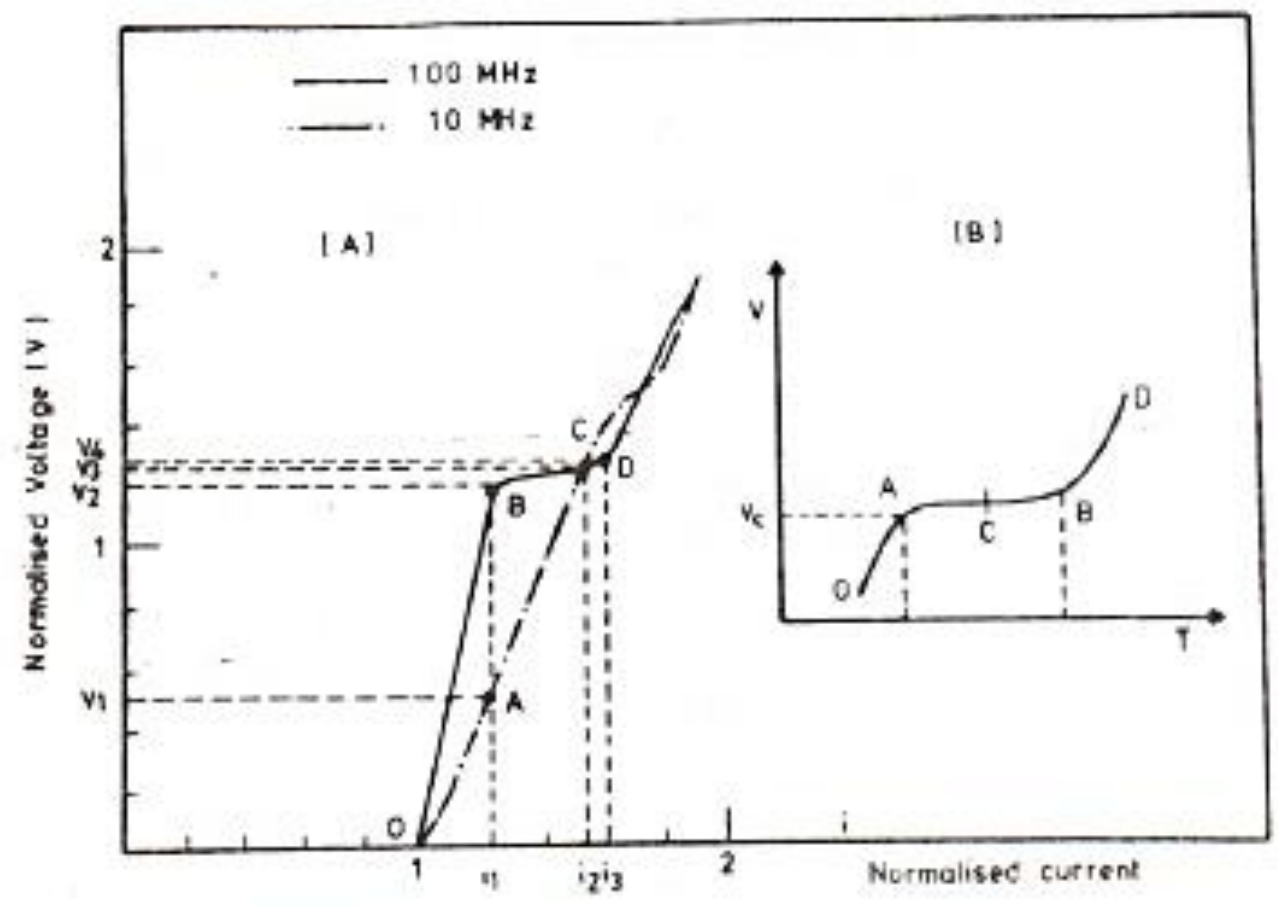

Figure (3): Comparison between $\mathrm{I}-\mathrm{V}$ curves for if frequencies 10 and $100 \mathrm{MHz}$ The effect of the if frequency is clesrly shown on the shatpening of the tirst step.

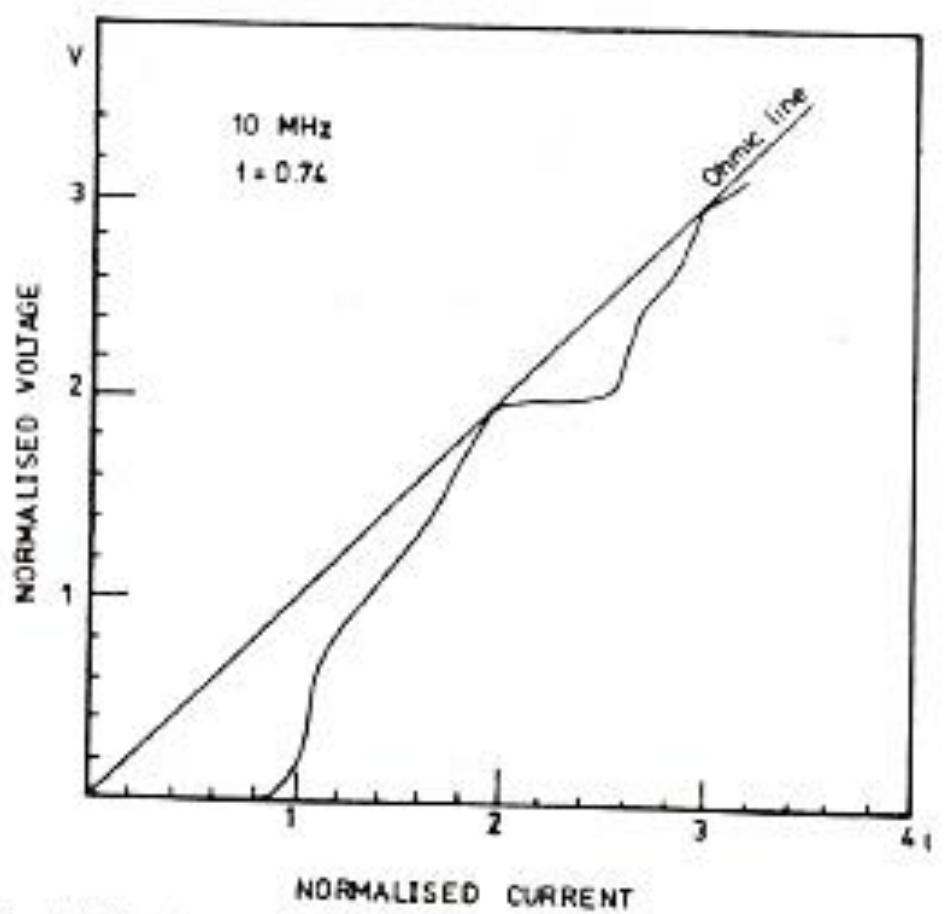

Figure (4): Calculeted $\mathrm{I-V}$ curve for an $\mathrm{t}$ frequency $10 \mathrm{MHz}$ and notmalized
temperature $\mathrm{t}=.74$. 
Now since curve II is at a higher frequency, thes there are thore if cycles irrvalved than that for curve 1 during the same range of current. Mote if cycles means that the winding process is much greatet, hence a higher phase transitian rate is expected for curve II, which leads to the fact that $v_{2}>v_{1}$. Thus we see thet as the $\mathrm{If}$ frequency is increased, the step rise (OB) sharpens. On the ather hand tor curve 1, we see that an further increasing the curtent, it will rise to point $C$, beyond which a stcp is observed at $v \sim 1.5,1 \sim 1.5$ which is a subhatmonic step $(n=3 / 2)$. Now back to curve $\mathrm{B}$, on increasing the current beyond point $\mathrm{B}$, a step width is observed. Fram B to $C$, the transition rate increases untill at point $C$ we have a single phase tranaition every complete it cycle. I1 our if curtent was not rectified, we would then have the step extended $101 \sim 2$ where two trarsitiona occurs every of cycle. But since we have a rectified curtent the step width will tetminate at point $D$ and a new riset stats again leading to the sacand step. Thus from the figure and our discussion our wotk suggest that at low if frequency there is a step (second subharmonic), and as the $t f$ frequency is increased the strp is shifted downwards untill it completly assumes the position of the first fundamnatal step with a width equal to half the eritical current.

In the second region, the model shuws steps existing at $U \sim 2,2.5$ and $\sim 3$, in othet words we have fundamental and subhatmonic steps. Such behavior is due to the nature of the $t 1$ cutrent considered in this wark. The general features of these steps ere the same as discussed elsewhere, hence there is no need ta repeat them sgoin. It is wosth noting that aatier, we mentioned that the effect of varying the If frequency and keeping the modulation trequency, is the same if the opposite is done. Thus our work predicts that by changing the modusation frequency and keeping the junction at a constant if frequency we would be able to observe such phenomeno. In order to more test our model, and get an extra-support for our work we have alsa solved the equations for a normalized temperature $t=.74$ (i.e $\left.i_{N}>0\right)$ and an sf frequency $10 \mathrm{MHz}$, with no change in the modulation frequency. The results are shown in figure (4) where we notice the following. The sccond subhatmonic step is completely washed out, while that for the third it is less pronounced than that for $T=0$. The fundamental steps show a slight rounding on their knees. Hence the effect of thermal current is only vrry strong an subtrarmonic steps, this is in agreement with ref (3).

\section{Conclusion:}

There afe several important conctusion as a result of aut work discussed sbove. First we have shown the effect of $\mathrm{ff}$ frequency on the evolution of the first step. Second, Dur wark praves that such eflect can also be observed by keeping the tI frequency constant, and varying the madulation frequency. Last, the rectified current cansidered in our work lesds to the eppearance of subharmaric ateps. This last result suygest a new mean of experimentally detecting subharmoric ateps. 


\section{$\underline{\text { References: }}$}

1. C. Vanneste, A. Gilabert, P. Sibillot, and D.B. Ostrowsley. J. Low Temp. Phy., 45, 517 (1981).

2. H. Seifert, J. Low Temp. Phys., 37, 595 (1979).

3. E. Ben Jacob and D.J. Bergman, Phys. Rev. A, 29, 2021 (1984).

4. T.M. Klapwijk, M. Sepers, and J.E. Moij. J. Low Temp. Phys., 27, 801 (1977).

5. L.D. Jackel and R.A. Buhrman. J. Low Temp. Phys., 19, 201 (1975).

6. Private Communications with Prof. R. Peters, CUA, Washington, D.C.

7. D.E. McCumber, J. Appl. Phys. 39, 3113 (1968). 